\title{
Desigualdades en Salud Bucal para Personas Mayores en Tiempos del COVID-19. La Teleodontología y la Odontología de Mínima Intervención como Caminos de Solución.
}

\section{Oral Health Inequalities for the Elderly in Times of COVID-19. Teledentistry and Minimal Intervention Dentistry as Solution Paths.}

\author{
Soraya León ${ }^{1,2,3}$, Rodrigo A. Giacaman $1,2,3^{*}$
}

1. Unidad de Odontogeriatría y Unidad de Cariología, Departamento de Rehabilitación Buco Máxilofacial, Facultad de Ciencias de la Salud,

Universidad de Talca, Talca, Chile.

2. Sociedad de Odontogeriatría de Chile.

3. Centro de Epidemiología y Vigilancia de las

Enfermedades Orales (CEVEO). Santiago, Chile.

* Correspondencia Autor: Prof. Rodrigo A. Giacaman, DDS, PhD. | Departamento de Rehabilitación Buco Máxilofacial, Facultad de Ciencias de la Salud, Universidad de Talca, Chile | Dirección: 1 Poniente 1141, Talca, Chile | Teléfono: 56-71-2201546 | E-mail: giacaman@utalca.cl

Trabajo recibido el 24/05/2019

Trabajo revisado 02/07/2020

Aprobado para su publicación el 12/07/2020
Se hace constar que en el manuscrito en el epígrafe de las páginas decía Giacaman R. y cols. y se hizo el cambio por León y Giacaman.

DOI: 10.4067/S2452-55882020000300147 